\title{
Raman Enhancement on a Broadband Meta-Surface
}

\author{
Sencer Ayas, ${ }^{\dagger, *}$ Hasan Güner, ${ }^{\dagger}$ Burak Türker, $^{\dagger}$ Okan Öner Ekiz, ${ }^{\dagger}$ Faruk Dirisaglik, ${ }^{\ddagger}$ Ali Kemal Okyay, ${ }^{\dagger, \S}$ and \\ Aykutlu Dâna ${ }^{\text {t,* }}$ \\ ${ }^{\dagger}$ UNAM Institute of Materials Science and Nanotechnology, Bilkent University, 06800 Ankara, Turkey, ${ }^{\ddagger}$ Department of Electrical Engineering, University of \\ Connecticut, Storrs, Connecticut 06269, United States, and ${ }^{\S}$ Department of Electrical and Electronics Engineering, Bilkent University, 06800 Ankara, Turkey
}

$\mathrm{P}$ lasmonic excitations of metallic nanostructures have attracted a great deal of attention in past decades, due to the rich variety of geometric configurations, the associated optical properties and phenomena, and the wide range of present and potential future applications. ${ }^{1,2}$ Propagating and localized plasmons have been utilized in the design of photonic structures to efficiently couple free-space propagating light onto highly confined surface modes, resulting in the enhancement of electromagnetic field intensities. Nonlinear optical effects benefit from plasmonic field enhancement, ${ }^{3,4}$ and plasmonics has the potential to be an enabling technology for quantum optics and all-optical information processing. ${ }^{5,6}$ It has been shown that plasmonic field enhancement allows the observation of Raman scattering from single molecules with low excitation powers down to microwatts. ${ }^{7,8}$ The lack of reliability resulting from the spatially non-uniform nature of plasmonic field enhancement can be a problem for applications requiring repeatability. In the case of surfaceenhanced Raman scattering (SERS), regions with high enhancement (so-called hot spots) are typically major contributors to the observed signal. Raman intensity enhancement is estimated through $I_{\text {SERS }} \cong$ $I_{0}\left|E\left(\omega_{\text {exc }}\right) E\left(\omega_{\text {det }}\right) / E_{0}\left(\omega_{\text {exc }}\right) E_{0}\left(\omega_{\text {det }}\right)\right|^{2}$, where $\omega_{\text {exc }}$ and $\omega_{\text {det }}$ are the excitation and detection frequencies, and $E$ and $E_{0}$ are the electric field intensities with and without the presence of plasmonic structures. Defining an enhancement factor, $E F(\omega)=\left|E(\omega) / E_{0}(\omega)\right|^{2}$, overall Raman enhancement factor can be written as the product of excitation and detection factors, $E F_{\text {SERS }}=E F\left(\omega_{\text {exc }}\right) E F\left(\omega_{\text {det }}\right)$. Spatial nonuniformity of the electric field directly translates into a spatial non-uniformity of $E F_{\text {SERS }}$ and can be an important disadvantage for repeatability. Hot spots are typically formed when two metal regions come close (within a

\section{ABSTRACT}
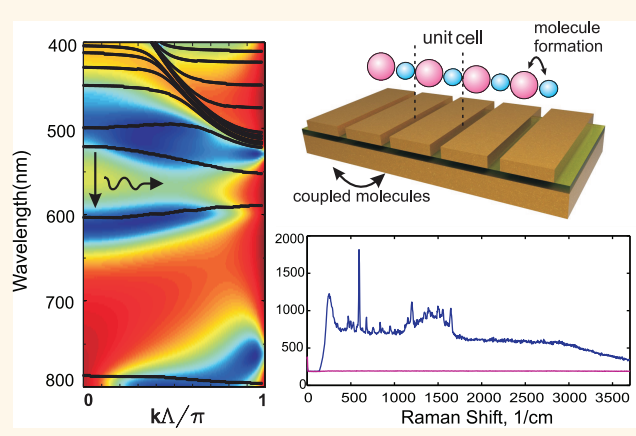

Plasmonic metamaterials allow confinement of light to deep subwavelength dimensions, while allowing for the tailoring of dispersion and electromagnetic mode density to enhance specific photonic properties. Optical resonances of plasmonic molecules have been extensively investigated; however, benefits of strong coupling of dimers have been overlooked. Here, we construct a plasmonic meta-surface through coupling of diatomic plasmonic molecules which contain a heavy and light meta-atom. Presence and coupling of two distinct types of localized modes in the plasmonic molecule allow formation and engineering of a rich band structure in a seemingly simple and common geometry, resulting in a broadband and quasi-omni-directional meta-surface. Surfaceenhanced Raman scattering benefits from the simultaneous presence of plasmonic resonances at the excitation and scattering frequencies, and by proper design of the band structure to satisfy this condition, highly repeatable and spatially uniform Raman enhancement is demonstrated. On the basis of calculations of the field enhancement distribution within a unit cell, spatial uniformity of the enhancement at the nanoscale is discussed. Raman scattering constitutes an example of nonlinear optical processes, where the wavelength conversion during scattering may be viewed as a photonic transition between the bands of the meta-material.

KEYWORDS: plasmonics · metamaterials · surface-enhanced Raman spectroscopy $\cdot$ coupled plasmonic modes

few nanometers) to each other, and even periodic structures may display a wide distribution of enhancement factors. ${ }^{9}$ In order to achieve high and spatially uniform field enhancement, engineered surfaces that exhibit plasmon modes at both the excitation and scattering wavelengths are needed. ${ }^{10-13}$ Previously, metal nanoparticle clusters (bottom-up approach) and sparse structures or biharmonic gratings with

\begin{abstract}
* Address correspondence to ayas@bilkent.edu.tr, aykutlu@unam.bilkent.edu.tr.
\end{abstract}

Received for review April 16, 2012 and accepted July 30, 2012.

Published online July 30, 2012 10.1021/nn301665a

C 2012 American Chemical Society 


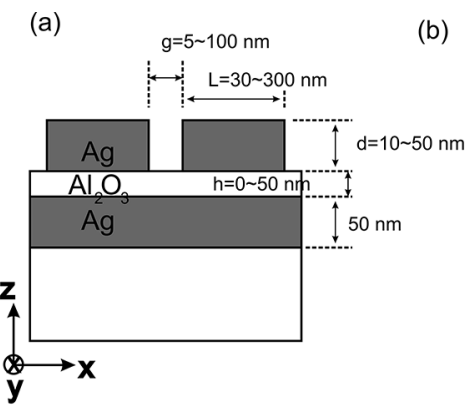

(c)

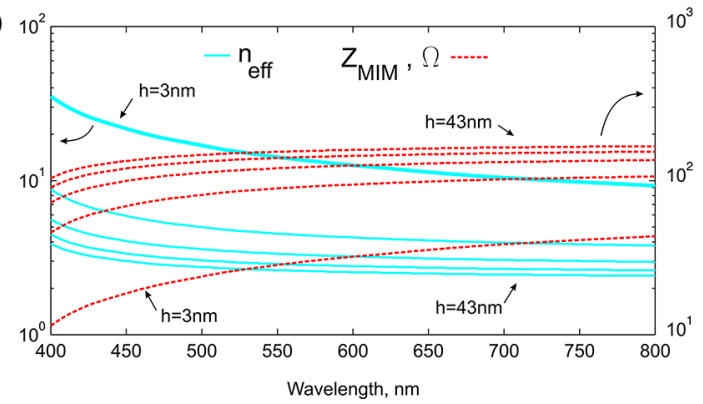

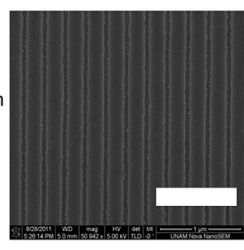

(d)

(e)
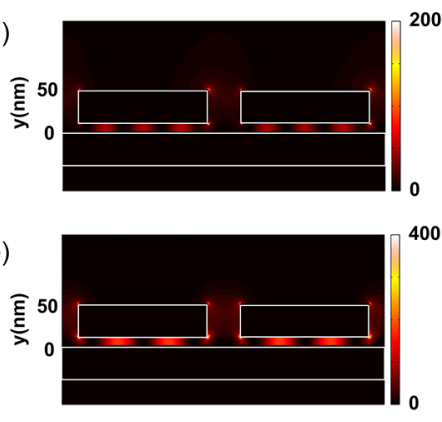

(f)

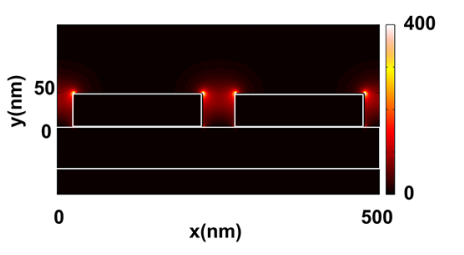

(g)

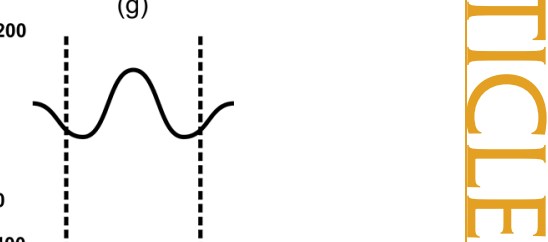

Figure 1. (a) Schematic cross section of metal-insulator-metal (MIM) meta-material structures. The metal layers are evaporated $\mathrm{Ag}$, and the dielectric layer $\left(0-40 \mathrm{~nm}\right.$ thick) is $\mathrm{Al}_{2} \mathrm{O}_{3}$ deposited. (b) Scanning electron micrograph of a representative structure is shown; scale bar $1 \mu \mathrm{m}$. (c) Effective index ( $\left.n_{\text {eff }}\right)$ and impedance $\left(z_{\text {MIM }}\right)$ of the MIM fundamental TM mode as a function of wavelength for various dielectric layer thicknesses $(3,13,23,33$, and $43 \mathrm{~nm})$ are given for convenience. (d) Depending on the frequency of excitation, different resonant modes can be excited within the MIM section. In the one-dimensional case, the MIM can be viewed as a transmission line, whose propagation constant (or effective index) depends on the gap between the top and bottom metal layers. Truncation at both ends results in a Fabry-Perot (FP)-type resonator. Electric field intensity squared is plotted for a $200 \mathrm{~nm}$ long MIM structure with $10 \mathrm{~nm}$ dielectric gap excited at $555 \mathrm{~nm}$, corresponding to a resonance of the FP resonator. (e) When the same structure is excited at $660 \mathrm{~nm}$, another resonance is excited displaying a different field distribution. (f) When the gap is reduced to zero, one resonant mode persists in the vertical transmission line cavity formed with side walls of the top metal layer as conducting planes. (g) Cartoons show the field amplitudes of modes shown in (d, e, and $f$ ).

double resonances (top-down approach) were used for this purpose. ${ }^{14}$ Bottom-up approach substrates offer the advantage of simplicity and offer uniformity over large scales; on the other hand, the statistical nature of production processes, in principle, prevents completely hot-spot-free and uniform enhancement on a microscopic scale. Sparse arrays of multiply sized nanoantennas, or multiperiodic structures, may be designed to enhance fields at both excitation and scattering frequencies, however, exhibit spatially non-uniform spectral properties over the extent of a wavelength. In contrast, plasmonic meta-materials possess subwavelength periodicity. When fabricated using a top-down approach, they have the potential to offer spatially uniform, wide band coupling required for uniform Raman enhancement.

In this article, we discuss the application of plasmonic meta-surfaces to SERS. We describe the resonances and field enhancements of a closely packed metalinsulator-metal configuration, which we refer to as multiply coupled plasmonic meta-material (MCPM). Strong coupling of the modes is shown to be effective for a subset of geometric parameters. Despite the seemingly common and simple geometry, we show that, under strong coupling of various types of modes, a rich band structure can be engineered within a wide spectral range, allowing highly controllable field enhancement at excitation and scattering frequencies, thereby allowing highly repeatable SERS.

Description of the Plasmonic Meta-Surface. A crosssectional schematic of the one-dimensional periodic MIM structure is shown in Figure 1a (a representative SEM micrograph is given in Figure 1b). MIM elements with relatively thick top metal layers $(c a .50 \mathrm{~nm}$ ), with widths of $150-200 \mathrm{~nm}$, are repeated with periods of 200 to $300 \mathrm{~nm}$, forming an inter-MIM spacing of $10-80 \mathrm{~nm}$. MIM structures can be viewed as nanoscale planar waveguides, ${ }^{15}$ and individual MIM elements have modes confined inside the dielectric region (referred to as waveguide mode). Localized resonances result from the Fabry-Perot (FP)-type resonator formed within the MIM waveguide, terminated at both ends. The propagation wavevector $\beta_{\mathrm{SPP}}$ and effective index $n_{\mathrm{eff}}=\beta_{\mathrm{SPP}} / k_{0}$ of the fundamental TM mode of a MIM waveguide can be calculated by solving for $\beta_{\text {SPP }}$ in the following equation ${ }^{15}$

$$
k_{\mathrm{m}} \varepsilon_{\mathrm{d}}+k_{\mathrm{d}} \varepsilon_{\mathrm{m}} \tanh \frac{k_{\mathrm{d}} w}{2}=0
$$

where $k_{\mathrm{d}}=\left(\beta_{\mathrm{SPP}}^{2}-\varepsilon_{\mathrm{d}} k_{0}^{2}\right)^{1 / 2}, k_{\mathrm{m}}=\left(\beta_{\mathrm{SPP}}^{2}-\varepsilon_{\mathrm{m}} k_{0}^{2}\right)^{1 / 2}$, and $k_{0}=2 \pi / \lambda$ is the propagation wave vector in free space for wavelength $\lambda$. Typically, $\beta_{\text {SPP }}$ is a complex number, and the real part of $\beta_{\text {SPP }}$ can be used to infer the effective wavelength. Assuming a unity relative magnetic 
permeability for propagating TM modes inside the MIM waveguide, the effective index ( $n_{\text {eff }}$ ) can also be used to estimate the impedance of the transmission line, $z_{\text {MIM }}=$ $120 \pi / n_{\text {eff }}$ (see Figure 1c). The propagation constant calculated using eq 1 can be used to estimate the resonances of a FP cavity. The roundtrip phase is written as $\varphi(\lambda)=2 \beta_{\mathrm{SPP}} w+2 \Delta \varphi(\lambda)$, where $2 \beta_{\mathrm{SPP}} w$ is the phase shift due to propagation, and $2 \Delta \varphi(\lambda)$ is the total phase shift acquired upon reflection at the ends of the resonator. A FP resonator exhibits resonances when $\mid 1-R_{0} \exp i \varphi$ $(\lambda)$ is a minimum, where $R_{0}$ is the magnitude of the reflection coefficient. Using the above formulation, absorption resonances of uncoupled MIM waveguides have been calculated. In general, $\Delta \varphi(\lambda)$ may be calculated through the reflection coefficient at the end of the waveguide, taking into account the impedance mismatch between the waveguide mode and free-space modes. ${ }^{16}$

Optical properties of arbitrary metallic nanostructures have been commonly analyzed using optical first-principles calculations. Optical properties of simple geometric configurations, on the other hand, can be explained in terms of lumped circuit elements. ${ }^{17-21}$ The waveguide perspective is practically adequate, as the experimentally observed optical properties of isolated structures can be well explained using this approach. ${ }^{22}$ Figure $1 \mathrm{~d}$,e shows the electric field profiles when such structures are excited at two different wavelengths for a dielectric thickness of $12 \mathrm{~nm}$. Standing wave patterns within the MIM are observed when the surface is excited near its resonances, validating the FP resonator approach (cartoons in Figure 1g show E-field along the structure for various modes in Figure $1 d-f$ ).

In the one-dimensional case, when the dielectric thickness is reduced to zero, the MIM waveguide modes disappear; however, the remaining structure still exhibits a localized mode (Figure 1f). This remaining vertical cavity mode (referred to as LFP, short for localized FabryPerot) is formed due to FP-type resonance in the vertical metallic cavity between the top metal layers of consecutive MIM regions. ${ }^{18}$ The resonance frequency of the LFP can be approximately calculated using lumped models or using the waveguide approach used above. The fundamental resonance of closely spaced MIM structures also have been modeled previously using lumped LC elements. ${ }^{18,23}$ Using such a model, the LFP mode frequency can be approximated by $\omega_{\text {LFP }}=\left(\left(C_{\mathrm{e}}+C_{\mathrm{m}}+\left(C_{\mathrm{m}}^{2}+C_{\mathrm{e}}^{2}\right)^{1 / 2}\right) /\left(L C_{\mathrm{m}} C_{\mathrm{e}}\right)\right)^{1 / 2}$, where $L \cong$ $0.5 \mu h w+3 w /\left(2 \varepsilon_{\mathrm{O}} h w_{\mathrm{P}}^{2}\right)$ is the inductance per unit width ( $\mu$ is the magnetic permeability, $h$ is the dielectric thickness, and $w$ is the width of the MIM), $C_{\mathrm{e}} \cong c \varepsilon_{\mathrm{O}} h / g$ is the capacitance per unit width due to coupling between MIM top metals ( $\varepsilon$ is the dielectric constant, $h$ is the dielectric thickness, $g$ is the gap between MIMs, and $c$ is a correction parameter), $C_{\mathrm{m}} \cong 0.25 \varepsilon_{\mathrm{O}} \varepsilon h / g$ is the capacitance per unit width due to coupling of top metal layer of MIMs to the metallic ground plane. The model ignores resistive losses.
When the areal density of such structures is increased to improve surface coverage, strong coupling of individual structures becomes inevitable, modifying the spectral properties, as well as the band structure.

Coupled Meta-Atoms and Meta-Molecules. The LFP mode can be considered as a light meta-atom, possessing a single energy level (Figure 2a). Similarly, the MIM structure constitutes a heavy meta-atom, with more than one energy level (Figure $2 \mathrm{~b}$ ). When the atoms are brought into contact by a coupling mechanism, bands emerge similar to the coupled resonator optical waveguide $(\mathrm{CROW}){ }^{24} \mathrm{~A}$ periodic arrangement of grooves (i.e., LFPs) results in a monatomic crystal (Figure $2 \mathrm{c}$ ). In the monatomic case, coupling is primarily through the surface plasmon mode (SPP). Collective excitations in nanoparticle arrays have been previously reported, and for large interparticle spacing, weak coupling occurs through radiative routes. ${ }^{25-27}$ On the other hand, when MIMs are arranged in a closely packed periodic fashion, MIM modes and the LFP modes strongly couple due to overlapping modes, rather than due to radiative coupling, resulting in a diatomic crystal (Figure $2 \mathrm{~d}$ ). The coupled MIM waveguides could also be viewed to be similar to a resonant guided wave network (RGWN); ${ }^{28}$ however, coupling of waveguides through an intermediate localized resonance in the vertical cavity distinguishes the MCMP from the RGWN.

The coupling of the MIM and LFP modes results in an anticrossing behavior, as shown in Figure 2e,f. In numerical calculations, it is seen that anti-crossing-over behavior due to coupling of MIM modes with the LFP (around $h \sim 17 \mathrm{~nm}$ in Figure 2e,f) leads to an improvement of the absorption bandwidth.

The angular dependence of coupling to the resonances is best visualized in the band structure of the plasmonic meta-surface. In order to intuitively understand the dependence of the band structure on design parameters, we construct a circuit model, as shown in Figure $2 \mathrm{~g}$. The MIM section is modeled using a transmission line, and the LFP is modeled using an LC resonator. A capacitor is used to model the coupling. The onedimensional band structure is calculated by constructing an $A B C D$ matrix for the lumped element model of the unit cell. In order to obtain the band structure, the ratio of the input and output parameters of the $A B C D$ matrix are assumed to be $\exp (i k \Lambda)$ for a given propagation vector $k$ and period $\Lambda$. In order to find the resonance frequencies, one needs to solve for $\omega$ through

$$
\left|I-\left[\begin{array}{ll}
A & B \\
C & D
\end{array}\right] e^{i k \Lambda}\right|=0
$$

where $l$ is the $2 \times 2$ identity matrix.

The $A B C D$ matrix of the unit cell is constructed by multiplying $A B C D$ matrices for the transmission line representing the MIM section with $A B C D$ matrices for the lumped elements for coupling and LFP sections of 


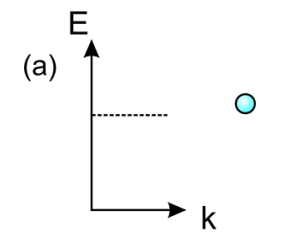

(b)

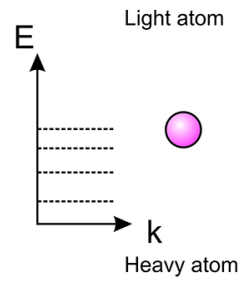

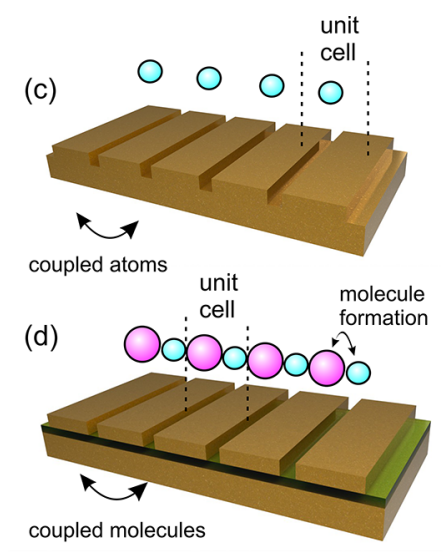

(g)

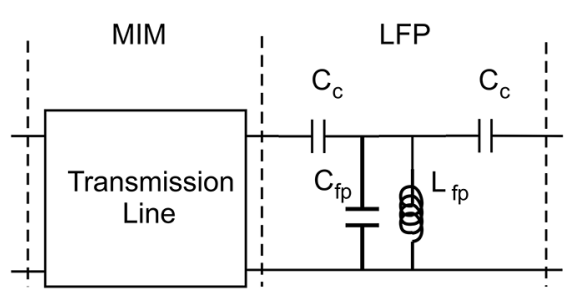

(e)

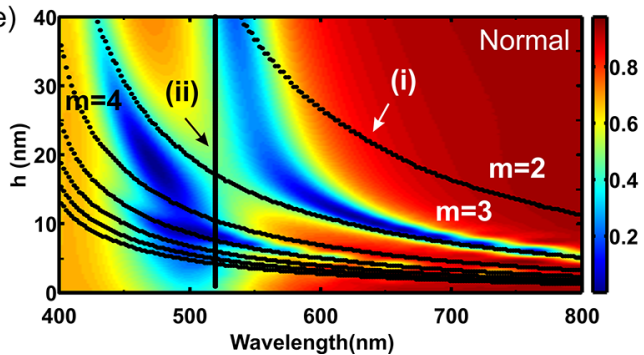

(f)

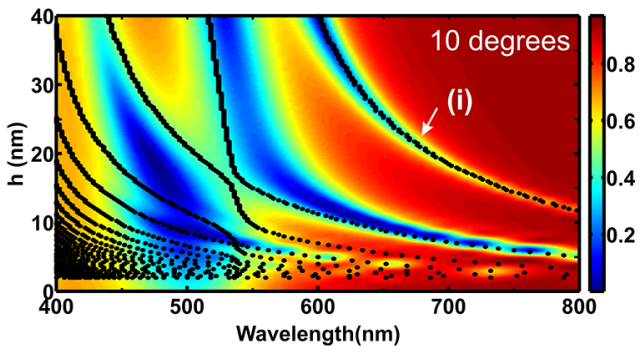

Figure 2. (a) Cavity resonance (LFP) can be thought as corresponding to a light atom, with a single localized state, and (b) MIM can be thought as corresponding to a heavy atom, with a multiple localized states. (c) In a one-dimensional periodic arrangement of the light atoms, coupling through the surface plasmon mode can introduce a coupling of the LFPs and propagating bands can be formed. (d) Similarly, in a one-dimensional periodic arrangement of the MIM structures with a relatively thick top metal and a thin gap dielectric, the MIM modes and the LFP mode formed between consecutive MIMs are hybridized to form a diatomic molecule. The molecules are coupled to form the band structure of the diatomic crystal. (e) Effect of mode coupling is observed in the reflection coefficient, plotted as a function of wavelength and dielectric thickness, $h$, for a one-dimensional crystal. Numerical computation results are shown for a $250 \mathrm{~nm}$ period structure with $180 \mathrm{~nm}$ MIM width, for normal incidence. Resonance wavelengths are calculated using the Fabry-Perot resonator model for the MIM structures (black lines; see text for details) that are superimposed. Antisymmetric modes (even mode number $m$ ) are not coupled to the free-space modes at normal incidence and, therefore, contribute no absorption (arrow (i)). The LFP mode wavelength is estimated using the LC model (vertical line around $520 \mathrm{~nm}$, denoted by arrow (ii)). (f) Reflection coefficient is plotted as a function of wavelength and dielectric thickness $h$ at a $10^{\circ}$ angle of incidence. Due to the broken symmetry, even modes also contribute to absorption. (g) Lumped circuit model for the diatomic unit cell consists of a transmission line capacitively coupled to a localized LC resonator representing the LFP mode. The model is used to calculate the resonance frequencies of the modes, plotted as dots, on top of the computational results in (f). The model captures the essential features of numerical calculations such as the resonance frequencies and anticrossing behavior due to coupling of modes.

the model. Resistive or radiative losses are ignored in the model. The $A B C D$ matrix for the transmission line section is given by

$$
A B C D_{\mathrm{TL}}=\left[\begin{array}{cc}
\cos (\beta I) & j z_{0} \sin (\beta l) \\
j \sin (\beta l) / z_{0} & \cos (\beta l)
\end{array}\right]
$$

The coupling capacitor has an impedance of $X_{c}=1 /\left(j \omega C_{\text {coupling }}\right)$. The $A B C D$ matrix for the coupling capacitor is given by

$$
A B C D_{\text {coupling }}=\left[\begin{array}{cc}
1 & X_{c} \\
0 & 1
\end{array}\right]
$$

The parallel LC resonator representing the localized Fabry-Perot (LFP) mode has an impedance $X_{\text {LFP }}$ given by $X_{\mathrm{LFP}}=1 /\left(1 /\left(j \omega L_{\mathrm{FP}}\right)+j \omega C_{\mathrm{FP}}\right)$. The ABCD matrix for the $\mathrm{LC}$ resonator representing the mode is given as

$$
A B C D_{\mathrm{LFP}}=\left[\begin{array}{cc}
1 & 0 \\
1 / X_{\mathrm{LFP}} & 1
\end{array}\right]
$$

The $A B C D$ matrix for the unit cell, $A B C D_{\mathrm{uc}}$ is calculated by multiplying the matrices in correct order, $A B C D_{\mathrm{uc}}=$ $A B C D_{\mathrm{TL}} \times A B C D_{\text {coupling }} \times A B C D_{\mathrm{LFP}} \times A B C D_{\text {coupling. }}$. The resonance frequencies calculated by the matrix method are shown on top of numerical results in Figure 2e,f. The model correctly captures the essential features of the numerical results, such as mode frequencies and anticrossing behavior.

In order to illustrate the effect of geometric parameters on the optical resonances and show the quasiomni-directional nature of the MCPM, we calculate the bands of several structures, as shown in Figure 3a-e. The monatomic crystal (no dielectric) is essentially a lamellar grating (cavity depth $50 \mathrm{~nm}$, width $50 \mathrm{~nm}$, period $250 \mathrm{~nm}$ ), and the LFP mode couples through the SPP to form a CROW-like band (Figure 3a). As the dielectric gap is introduced, MIM modes emerge in the reflection spectrum. For a thin dielectric of $h=3 \mathrm{~nm}$, a large number 

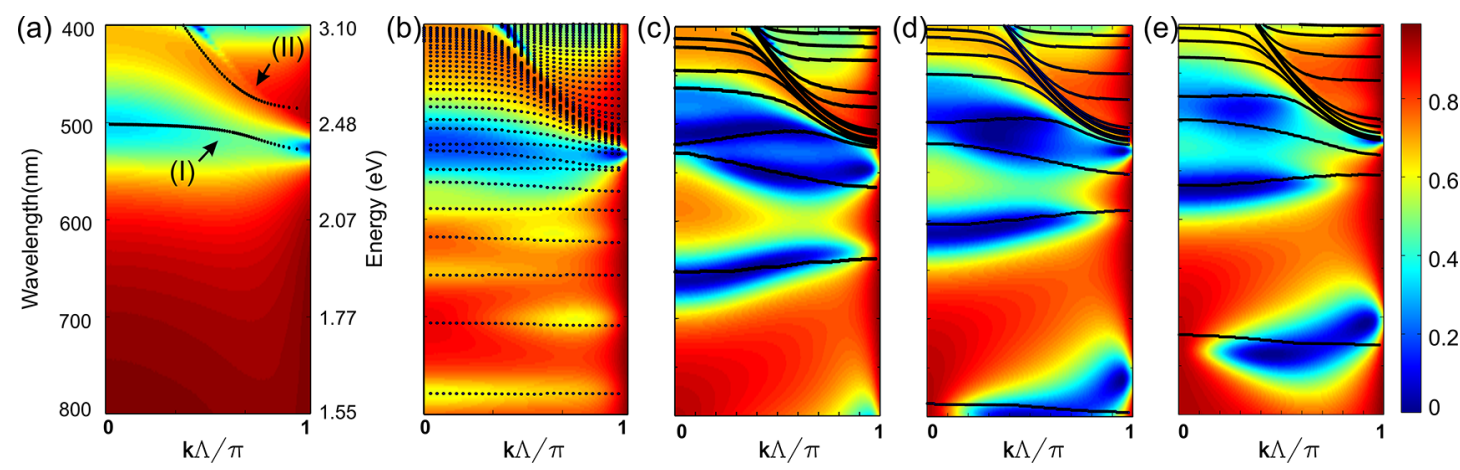

Figure 3. (a) Band structure of a monatomic meta-surface is calculated using the circuit model in Figure $2 \mathrm{~g}$ and the $A B C D$ matrix approach (see text) and superimposed on the reflection obtained through numerical calculations. When the dielectric thickness is zero, only the bands formed are through coupling of the LFP (arrow I) modes via the surface plasmonmode (arrow II) are observed. (b) Increasing the gap to $3 \mathrm{~nm}$ results in the appearance of a large number of MIM modes and formation of a diatomic metasurface. (c) Increasing the gap to $13 \mathrm{~nm}$ results in a reduction of the number of MIM modes, while increasing the absorption in the coupled bands due to improved impedance matching of the surface with free-space propagating modes. Further band structures are shown in (d) and (e) for 180 and $160 \mathrm{~nm}$ MIM widths, with $250 \mathrm{~nm}$ period. Color bar shows corresponding reflectance values.
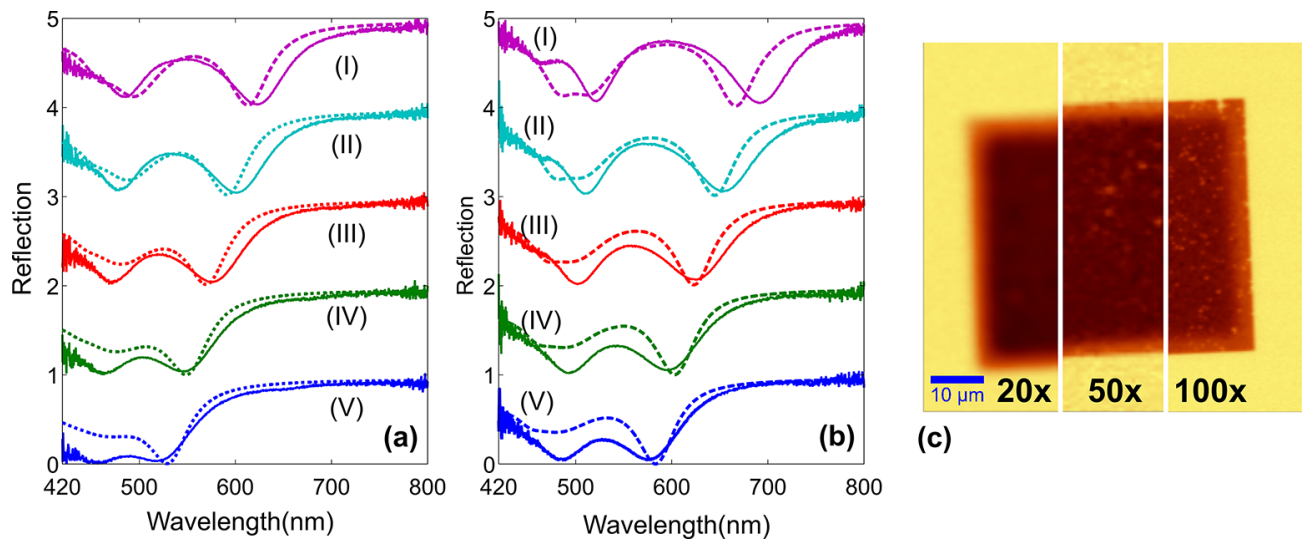

(c)

Figure 4. Theoretical and experimental reflectance plotted as a function of wavelength at normal incidence for (a) onedimensional, $250 \mathrm{~nm}$ period structures with nanowire widths of $180 \mathrm{~nm}$ (I), $170 \mathrm{~nm}$ (II), $160 \mathrm{~nm}$ (III), $150 \mathrm{~nm}$ (IV), $140 \mathrm{~nm}$ (V) and (b) one-dimensional, $300 \mathrm{~nm}$ period structures with nanowire widths of $200 \mathrm{~nm}$ (I), $190 \mathrm{~nm}$ (II), $180 \mathrm{~nm}$ (III), $170 \mathrm{~nm}$ (IV), $160 \mathrm{~nm}$ (V). (c) TM mode reflectance for a $10 \mathrm{~nm}$ band around $550 \mathrm{~nm}$ observed with different numerical apertures $(20 \times, 50 \times$, and $100 \times$ objectives) on one-dimensional $250 \mathrm{~nm}$ period $150 \mathrm{~nm}$ width structures. Insensitivity to numerical aperture demonstrates the quasi-omni-directional absorption of the surfaces.

of modes that span the visible spectrum emerge (Figure 3b). Consecutive bands with odd and even MIM modes show improved absorption at low and high angles due to their symmetry (a similar observation was reported for MIM resonator arrays in the terahertz frequency range ${ }^{16}$ ). The band structure, which is calculated by solving eq 2 , displays bands that coincide with reflection minima seen in numerical calculations. As the gap is increased to $12 \mathrm{~nm}$ (Figure $3 \mathrm{c}-\mathrm{e}$ ), the number of bands is reduced. The effect of the surface plasmon mode becomes visible at high frequencies and is included in band structure calculations as a perturbation (see Methods). The coupling of the SPP to the MIM/LFP structure has the effect of pushing and concentrating the bands to around $500 \mathrm{~nm}$ at high angles $(k \Lambda / \pi \sim 1)$ in Figure 3a-e.

\section{RESULTS}

The absorption spectra of the surfaces have been recorded at normal incidence using a low numerical aperture (NA $=0.05$ ) objective for both illumination and light collection. The reflection spectra are seen to agree with simulation results for various MIM widths and periods (Figure 4a,b). Evidence for the quasi-omnidirectional character of the one-dimensional structures can be seen in the high spatial resolution reflection maps shown in Figure 4c (average reflectance for a $10 \mathrm{~nm}$ spectral band centered at $550 \mathrm{~nm}$ is shown). When the structures are imaged by different numerical aperture lenses (objectives with $20 \times, 50 \times$, and $100 \times$ magnification and respective numerical apertures of $0.4,0.7$, and 0.95), the absorption is seen to be quite independent of the numerical aperture. This is in accordance with the expected quasi-omni-directional character of the band structures around $550 \mathrm{~nm}$.

For most applications in sensing and spectroscopy, the locations with high field enhancements must be exposed to allow for adsorption of molecules. Such regions are present in the MCPM (LFP region and top 
(a)
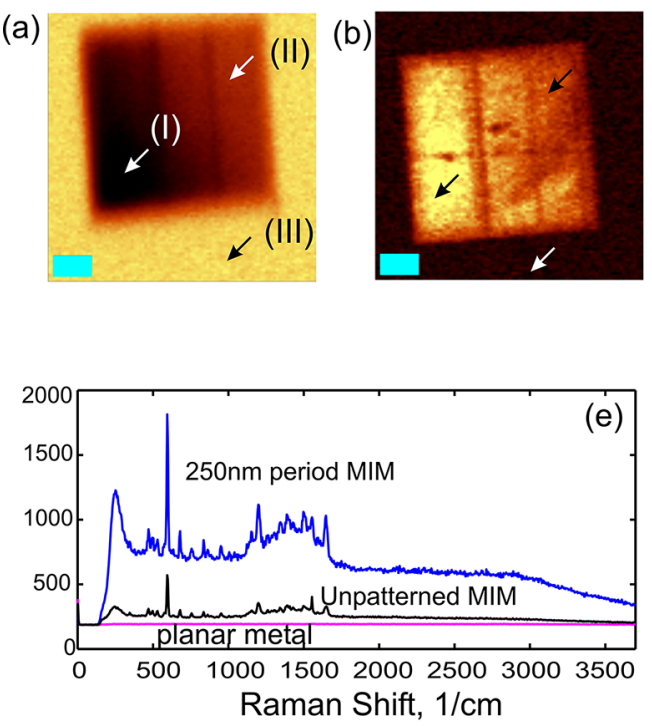
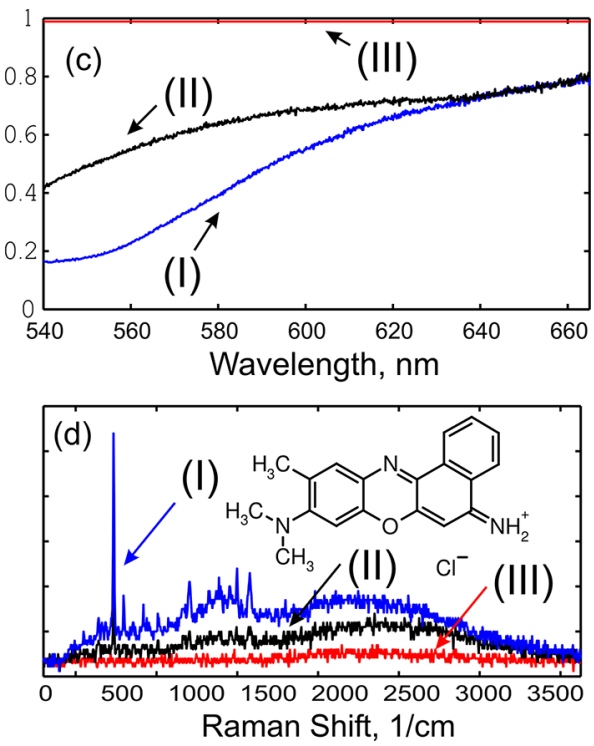

Figure 5. (a) Reflectance map (540-600 nm band) acquired using a $20 \times$ objective (NA 0.4 ) on a $250 \mathrm{~nm}$ period structure with $200 \mathrm{~nm}$ MIM width. (b) Raman spectra map (intensity of $591 \mathrm{~cm}^{-1}$ band, collected with $100 \mu \mathrm{W}$ excitation power at $532 \mathrm{~nm}$, $20 \times$ objective, $100 \mathrm{~ms}$ dwell time per pixel) of Cresyl Violet monolayer on the same structure. Scale bar is $10 \mu \mathrm{m}$. (c) Reflectance as a function of wavelength for several locations and (d) corresponding Raman spectra. Reflectance is plotted at two different locations (I) and (II) as referenced to location (III). Due to non-uniformity of the fabrication process, a gradient of the resonance wavelength is observed from top-right position to bottom-left position. When the absorption overlaps with excitation and emission wavelengths, improved Raman scattering is observed. Inset shows chemical structure of Cresyl Violet. (e) Raman signal is collected using a longer integration time (22 s, $100 \mu \mathrm{W}$ excitation power) on a planar silver surface, unpatterned MIM, and $250 \mathrm{~nm}$ period MIM regions. Although Cresyl Violet exhibits no Raman signal on the plane metal surface, some enhancement is seen on unpatterned MIM regions, possibly due to the surface roughness of the top layer.
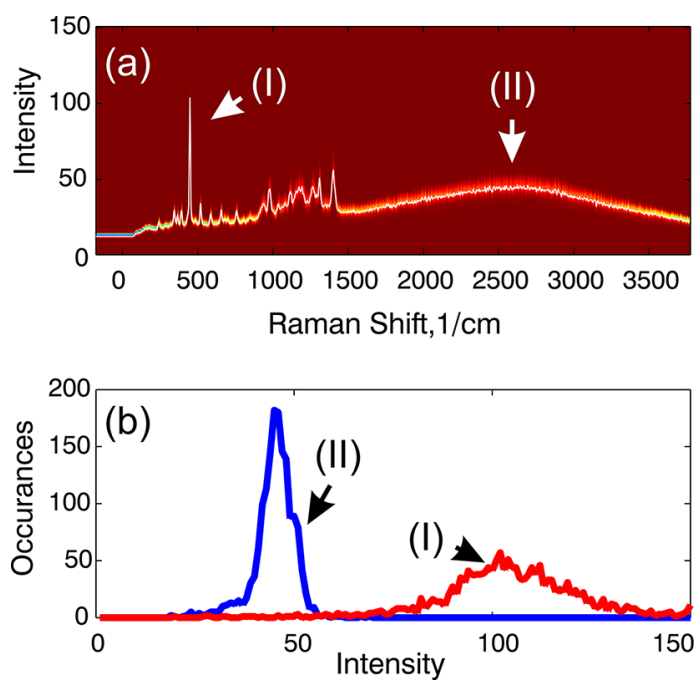

(c)

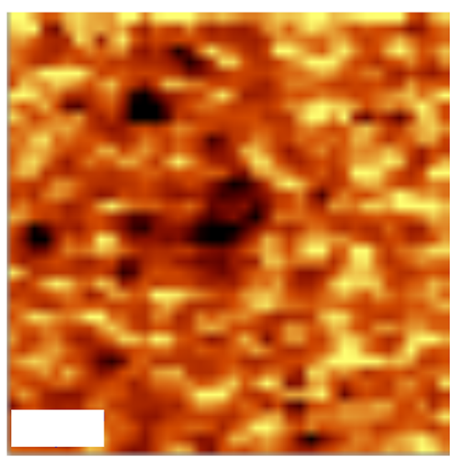

Figure 6. (a) Superimposed Raman spectra collected from 1600 individual spots over an area of $10 \mu \mathrm{m} \times 10 \mu \mathrm{m}$ (100 $\mu \mathrm{W}$ excitation power, $100 \times$ objective, and $\mathbf{4 0} \mathrm{ms}$ dwell time per pixel). (b) Histograms of intensity of two spectral locations shown by arrows (I) and (II) demonstrate uniform signal intensity within ca. $10 \%$ of average value for the fluorescence (arrow II) and ca. 20\% for the Raman signal (arrow I). (c) Raman map formed using the $591 \mathrm{~cm}^{-1}$ Raman band (scale bar $2 \mu \mathrm{m}$ ) where the contrast is enhanced to show several dead-spots with submicrometer dimensions, demonstrating the high-resolution imaging capability with such substrates.

surface), where the enhancement can be utilized for sensing or Raman spectroscopy. It should be noted that absorption and field enhancement are not necessarily proportional. However, numerical calculations show that if the resistive losses can be ignored, there is a strong correlation between the absorption and field enhancement. Typically, this is the case when the excitation frequency is reasonably below the plasma frequency of the metal and absorption is enhanced primarily due to plasmonic resonances. In our wavelength range of interest (400 $\mathrm{nm}$ to NIR), dielectric constant of silver allows this approximation. The MCPM can feature multiple absorption bands (and correlated field enhancement) that cover the entire excitation and scattering wavelengths (for example 250 period, $50 \mathrm{~nm}$ top metal, $12 \mathrm{~nm}$ dielectric thickness, and $200 \mathrm{~nm}$ top metal width). The advantage of the MCPM structures in plasmon-enhanced Raman 
(a)

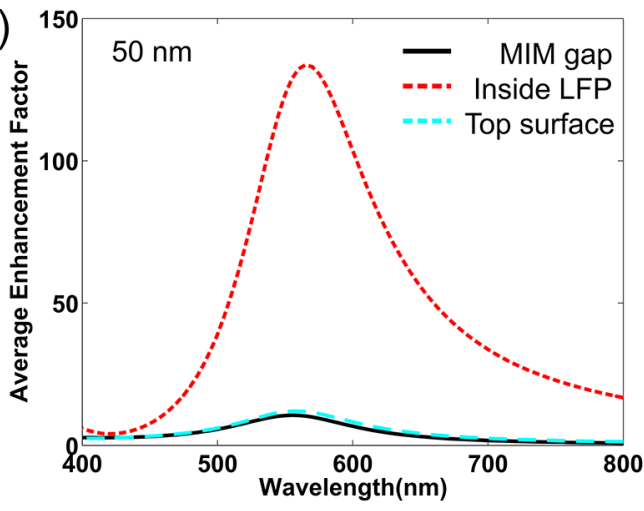

(c)

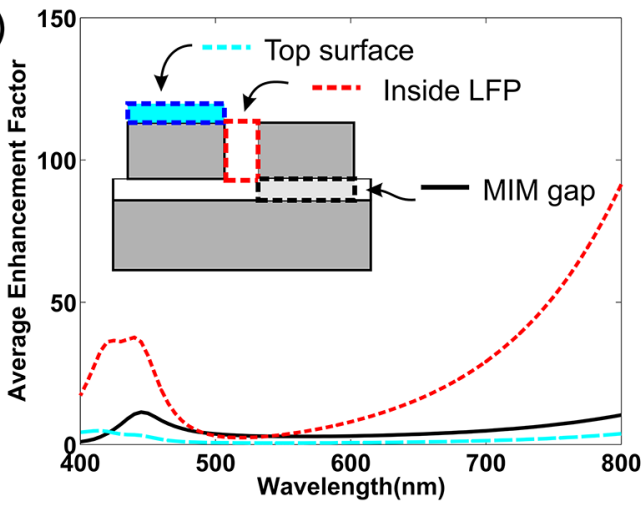

(e)

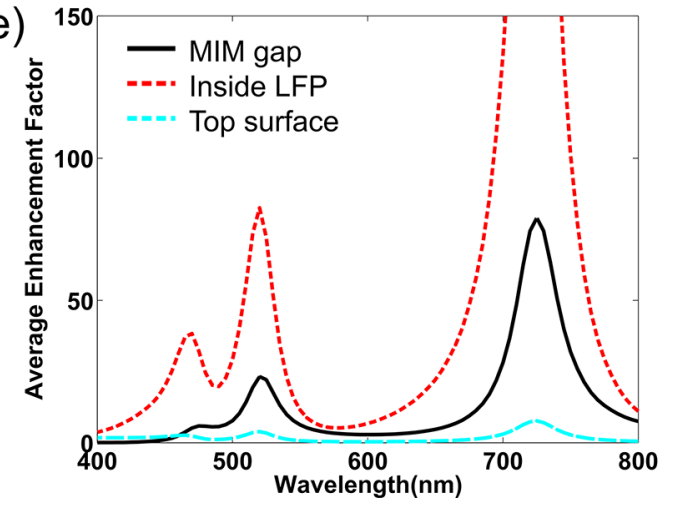

(b)

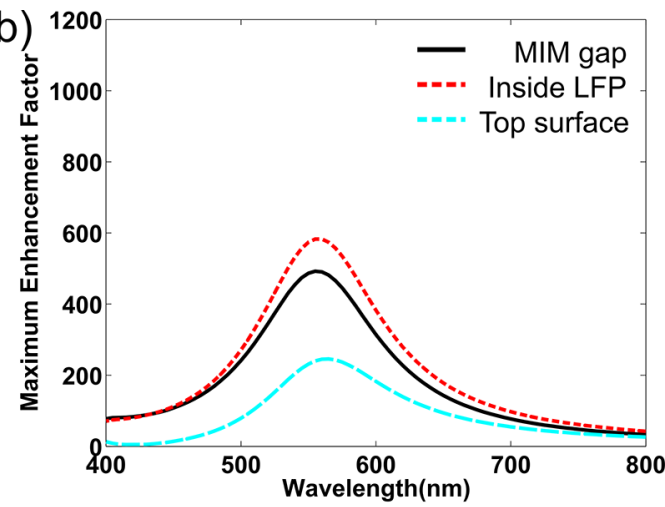

(d)

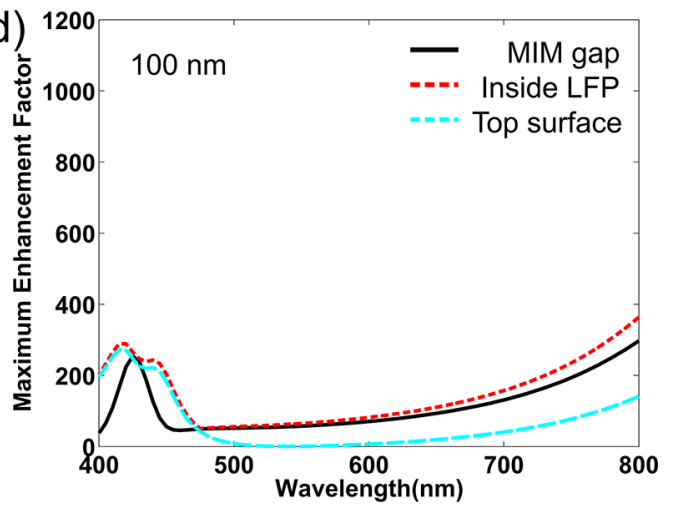

(f)

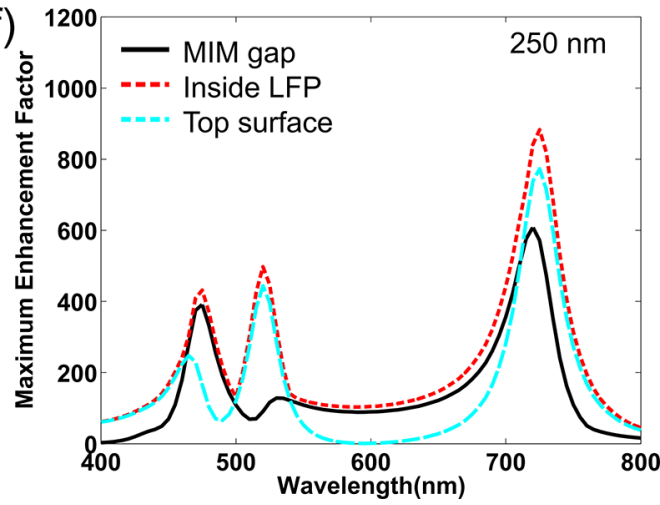

Figure 7. (a) Enhancement factors (EF) averaged over various regions (see inset in c) for a MIM structure with $50 \mathrm{~nm}$ periodicity, $10 \mathrm{~nm}$ top metal thickness, $20 \mathrm{~nm}$ dielectric gap, and $30 \mathrm{~nm}$ top metal width. (b) Maximum value of EF for different regions for the geometry in panel a. (c) Average EF values for a MIM structure with $100 \mathrm{~nm}$ periodicity, $20 \mathrm{~nm}$ top metal thickness, $20 \mathrm{~nm}$ dielectric gap, and $80 \mathrm{~nm}$ top metal width. (d) Maximum value of EF for different regions for the geometry in panel c. (e) Average EF values for a MIM structure with $250 \mathrm{~nm}$ periodicity, $50 \mathrm{~nm}$ top metal thickness, $20 \mathrm{~nm}$ dielectric gap, and $230 \mathrm{~nm}$ top metal width. (f) Maximum value of EF for different regions for the geometry in panel e. It is seen that shrinking the MIM size results in fewer resonances and improved average enhancement over the unit cell, especially on the top surface ( $10 \mathrm{~nm}$ thick slab over the top metal). The maximum values of the EFs are much higher than the average, showing the inherent spatial non-uniformity of enhancement. Comparing panels a-c, it is seen that tuning of the resonances through choice of geometry greatly improves $\mathrm{EF}$ for the wavelength range of interest (532 to $650 \mathrm{~nm}$ ).

spectroscopy is demonstrated using Cresyl Violet dye as the example molecule. Using low powers of about $100-300 \mu \mathrm{W}$ (measured by a placing a photodiode at the sample location in a separate measurement), the Raman spectra are recorded at various locations on the unpatterned MIM substrate (Figure 5a, arrow III) and on the MCMP (Figure 5a, arrows I and II). The unpatterned MIM reference produces little observable Raman or fluorescence signal, whereas the MCPM produces a pronounced enhancement, which is theoretically estimated to be on the order of $5 \times 10^{5}$ to $10^{6}$. The TM polarized reflectance $(10 \mathrm{~nm}$ band around $550 \mathrm{~nm}$, Figure 4 a) has a slightly non-uniform spectral response over a distance of about $50 \mu \mathrm{m}$, attributed to the exposure nonuniformity during the e-beam lithography. Raman signal map (591 $\mathrm{cm}^{-1}$ peak) collected at the same location is shown in Figure $5 \mathrm{~b}$. A spatial non-uniformity is also seen in the Raman signal (Figure 5b), correlated with 
the reflectance non-uniformity (Figure 5a). As expected, enhancement of the Raman signal is proportional to the absorption within the Raman band wavelength range (Figure $5 c, d$ ). When a longer averaging time of $22 \mathrm{~s}$ is used, still the Raman signal is absent on a flat Ag reference surface for the low power level used (Figure 5e). The fabrication related non-uniformity is insignificant over smaller length scales, and we superimpose the Raman spectra collected from 1600 locations within a $10 \mu \mathrm{m} \times$ $10 \mu \mathrm{m}$ square region in Figure $6 \mathrm{a}$. The fluorescence and Raman enhancements are uniformly enhanced (about $10 \%$ for fluorescence and about 20\% for Raman) over the region, as is seen in the histograms (Figure 6b) shown for two wavelengths. When the Raman signal is represented as an image map, several defects with submicrometer diameters are clearly resolved as dark spots in the image (Figure $6 \mathrm{c}$ ), indicating true highresolution imaging capability using the MCPM (image contrast is enhanced to clarify the dark spots).

Discussion of Spatial Uniformity of Enhancement at the Nanoscale. Due to the periodicity of the meta-surface, Raman enhancement appears to be uniform in the far field when the diffraction-limited spot size is larger than the meta-material period. However, it is important to understand the variations of local enhancement factor within a unit cell. We plot the average and maximum enhancement factors for three different geometries, as shown in Figure 7, for MIM periods of 50, 100, and $250 \mathrm{~nm}$. The averages are taken over rectangular sections over the top surface $(10 \mathrm{~nm}$ thick slab is chosen), within the LFP region (air gap region between the top metals) and inside the MIM gap, within the dielectric (see Figure 7c inset). The dielectric gap of the MIM structure does not contribute to the Raman signal since molecules cannot be placed there after fabrication. However, enhancement in this region may be important for other applications; therefore, we plot enhancement factors for this region for convenience. The correlation of EF values for the MIM and LFP regions also demonstrates the coupling of LFP and MIM resonances.

When comparing average and maximum EF of different regions and geometries, it is seen that for smaller structures ( $50 \mathrm{~nm}$ period, $30 \mathrm{~nm}$ width, Figure $7 \mathrm{a}$ ), a single resonance is present in the wavelength range of interest
(532 to $640 \mathrm{~nm}$ ), and average field enhancements at the top surface are greatly improved compared to $100 \mathrm{~nm}$ structures (Figure 7c) and $250 \mathrm{~nm}$ structures (Figure 7e). It is also seen that the LFP region has a stronger average enhancement factor as compared to the top surface and MIM gap. The maximum enhancement factors can be relatively large $\left(\mathrm{EF}_{\mathrm{exc}} \sim 1000, \mathrm{EF}_{\mathrm{SERS}} \sim 10^{6}\right)$ for both small and larger periods (Figure $7 b, d, f)$. From the data, it can be concluded that, despite the apparent uniform enhancement in diffraction-limited far field measurements, enhancements are mostly localized to the LFP region for larger structures. For smaller structures ( $\sim 50 \mathrm{~nm}$ period), although there is local nonuniformity and the LFP region still contributes the greater portion to the enhanced signal, the average EF values for the top surface and the LFP region have improved ratio, indicating improved uniformity.

\section{CONCLUSIONS}

The MCPM features propagation of multiple plasmon modes in the visible range of the spectrum, through direct capacitive coupling or coupling via a localized resonant mode. The simplicity of the MCPM allows fabrication of an ultrathin meta-surface with tailorable bands at wavelengths toward the near-UV. Bandwidths are comparable to thicker wideband absorbers. ${ }^{29}$ Due to the quasi-omni-directional coupling of light to the plasmon modes, high spatial resolution SERS imaging is possible. The diffractionlimited spot size is greater than the period of the metamaterial, and plasmonic enhancement of the Raman signal essentially occurs with a unity surface fill factor. High-resolution imaging capability can potentially improve biomolecular sensing. ${ }^{30,31}$ Raman excitation wavelength and scattering wavelengths coincide with different bands. Advancing the analogy of the meta-material with a semiconducting crystal, the Raman transition can be regarded as a photonic transition between two bands, where the transition is accompanied with the release of a phonon. Raman scattering constitutes an example for nonlinear optical phenomena, and in general, the MCPM can find application in cases where enhanced nonlinearity at low optical powers is desirable, such as enhancement of photonic interactions in all-optical classical or quantum information processing.

\section{METHODS}

Fabrication of Plasmonic Structures. Germanium $(2-3 \mathrm{~nm}$, $99.99 \%$ purity) is deposited on 2 by $2 \mathrm{~cm}^{2}$ silicon substrates using e-beam evaporation system (Vaksis PVD Vapor 4S e-beam) as wetting and adhesion layer with a deposition rate $0.5 \AA / \mathrm{s}$. Then $70 \mathrm{~nm}$ silver (99.99\% Purity) is deposited with e-beam system with a deposition rate of $0.6-0.8 \AA / \mathrm{s}$. For the dielectric spacer layer, $12 \mathrm{~nm} \mathrm{Al}_{2} \mathrm{O}_{3}$ (99.99\% Purity) is also deposited with the e-beam system with a deposition rate $0.3-0.4 \AA / \mathrm{s}$.

PMMA (MicroChem Nano 950K A2: $2 \%$ solid PMMA) is spincoated on the 2 by $2 \mathrm{~cm}^{2}$ silicon substrate that is coated with germanium, silver, and $\mathrm{Al}_{2} \mathrm{O}_{3}$ previously. The PMMA resistcoated samples are prebaked at $180{ }^{\circ} \mathrm{C}$ for 90 s. E-beam lithography is performed with FEI Nova NanoSEM equipped with Raith ElphyPlus system. The acceleration voltage is $30 \mathrm{kV}$, and the beam current is $26 \mathrm{pA}$. The MIM structures with widths changing between 100 and $200 \mathrm{~nm}$ are defined by varying the exposure dose. The areas of patterned regions are 50 by $50 \mu \mathrm{m}^{2}$. After the e-beam lithography step, $50 \mathrm{~nm}$ of silver is deposited on the samples using an e-beam evaporation system. After metal deposition, samples are placed in the $45^{\circ} \mathrm{C}$ semiconductor grade ethanol for $1 \mathrm{~h}$, which is followed by ultrasonic bath for 
$30 \mathrm{~s}$. Then the samples are taken from the ethanol and washed with isopropyl alcohol and deionized water. The samples are dried under nitrogen flow.

Optical Measurements. Reflection mapping images are obtained with WITEC Alpha 300 S system with white light illumination. Reflection is referenced to unpatterned regions of the surface. A custom-designed reflection setup was used in wideband reflection measurements.

Raman Measurements. Samples are immersed in $10 \mu \mathrm{M}$ Cresyl Violet in ethanol solution for $2 \mathrm{~h}$, rinsed with ethanol several times, and blow-dried with nitrogen. SERS measurements are performed using WITEC Alpha 300S Raman module. A solidstate $532 \mathrm{~nm}$ wavelength laser is used for excitation in the Raman measurements. For Raman mapping measurements $20 \times, 50 \times$, and $100 \times$ objectives are used with integration times of 22-40 ms. For single Raman measurements, $20 \times$ objective, $25 \mathrm{~s}$ integration time, and a $100 \mu \mathrm{W}$ excitation power are used.

Simulations. Simulations are done with rigorously coupled wave analysis (RCWA) and FDTD methods. Details of the RCWA method is found in the literature. ${ }^{32}$ Field profiles are simulated using a commercial computational tool (Lumerical). Dielectric function of silver is obtained from the literature. Germanium wetting layer is neglected in the simulations. The refractive index of electron-beam-evaporated $\mathrm{Al}_{2} \mathrm{O}_{3}$ is measured experimentally using a J.A.Woolam V-VASE ellipsometer, and a constant refractive index of $n_{\mathrm{Al}_{2} \mathrm{O}_{3}}=1.6$ is used for the $\mathrm{Al}_{2} \mathrm{O}_{3}$ dielectric layer. The thickness of supporting $\mathrm{Ag}$ is $50-70 \mathrm{~nm}$, and transmission to the silicon substrate is less than $1 \%$ of incident light over the visible spectrum; therefore, only reflection is calculated. Absorption is approximated as $A=1-R$. Perfectly matched layer (PML) boundary condition is used in the $z$-direction, and Bloch boundary condition is used in the $x$-direction (along the period) of structures. Germanium wetting layer is neglected in the simulations. The mesh size is used in the simulations are $1 \mathrm{~nm}$ by $1 \mathrm{~nm}$ except the dielectric spacer layer, where $0.25 \mathrm{~nm}$ by $1 \mathrm{~nm}$ mesh size is used. Field profiles are calculated by illuminating structures with monochromatic plane wave corresponding to resonance wavelengths. The effect of the surface plasmon mode is included in the analytical band structure calculations as a perturbation. This is done by using a generic Lagrangian, which can be used to describe a plasmonic dimer modeled by two capacitively coupled $L C$ resonators $\mathscr{P}=1 / 2 L_{1} \dot{Q}_{1}^{2}+1 / 2 L_{2} \dot{Q}_{2}^{2}-$ $1 / 2 L_{1} \omega_{1}^{2} Q_{1}^{2}-1 / 2 L_{1} \omega_{2}^{2} Q_{2}^{2}-M_{\mathrm{e}} \omega_{1} \omega_{2} Q_{1} Q_{2}$, where $L_{1}$ and $L_{2}$ are the inductances, $Q_{1}$ and $Q_{2}$ are the charges, $\omega_{1}$ and $\omega_{2}$ are resonance frequencies of individual LC resonators, and $M_{e}$ is the electric coupling. ${ }^{33}$ Using the above Lagrangian, for each state $\left(\omega_{\mathrm{MM}}, k\right)$ of the band diagram and the corresponding SPP mode $\left(\omega_{\text {spp, }} k\right)$, we calculate perturbed frequencies $\omega_{\text {MM }}^{\prime}$ and $\omega_{\text {spp }}^{\prime}$ for the given wave vector $k$.

Conflict of Interest: The authors declare no competing financial interest.

Acknowledgment. This work was partially supported by TUBITAK under Grant 111M344, EU FP7:People-IAPP NanoBacterPhageSERS and the State Planning Organization of Republic of Turkey, project UNAM.

\section{REFERENCES AND NOTES}

1. Raether, H. Surface Plasmons; Springer-Verlag: Berlin, 1988.

2. Barnes, W. L.; Dereux, A.; Ebbesen, T. W. Surface Plasmon Subwavelength Optics. Nature 2003, 424, 824.

3. Popov, A. K.; Myslivets, S. A.; George, T. F.; Shalaev, V. M. Four-Wave Mixing, Quantum Control, and Compensating Losses in Doped Negative-Index Photonic Metamaterials. Opt. Lett. 2007, 32, 3044.

4. Palomba, S.; Zhang, S.; Park, Y.; Bartal, G.; Yin, X.; Zhang, X. Optical Negative Refraction by Four-Wave Mixing in Thin Metallic Nanostructures. Nat. Mater. 2012, 11, 34.

5. Choy, J. T.; Hausmann, B. J. M.; Babinec, T. M.; Bulu, I.; Khan, M.; Maletinsky, P.; Yacoby, A.; Lončar, M. Enhanced SinglePhoton Emission from a Diamond-Silver Aperture. Nat. Photonics 2011, 5, 738.

6. Chuang, I. L.; Yamamoto, Y. Simple Quantum Computer. Phys. Rev. A 1995, 52, 3489.
7. Kneipp, K.; Wang, Y.; Kneipp, H.; Perelman, L. T.; Itzkan, I.; Dasari, R. R.; Feld, M. S. Single Molecule Detection Using Surface-Enhanced Raman Scattering (SERS). Phys. Rev. Lett. 1997, 78, 1667.

8. Nie, S. Probing Single Molecules and Single Nanoparticles by Surface-Enhanced Raman Scattering. Science 1997, 275, 1102.

9. Fang, Y.; Seong, N.-H.; Dlott, D. D. Measurement of the Distribution of Site Enhancements in Surface-Enhanced Raman Scattering. Science 2008, 321, 388.

10. Baumberg, J. J.; Kelf, T. A.; Sugawara, Y.; Cintra, S.; Abdelsalam, M. E.; Bartlett, P. N.; Russell, A. E. Angle-Resolved SurfaceEnhanced Raman Scattering on Metallic Nanostructured Plasmonic Crystals. Nano Lett. 2005, 5, 2262.

11. Kocabas, A.; Ertas, G.; Senlik, S. S.; Aydinli, A. Plasmonic Band Gap Structures for Surface-Enhanced Raman Scattering. Opt. Express 2008, 16, 12469.

12. Banaee, M. G.; Crozier, K. B. Mixed Dimer Double-Resonance Substrates for Surface-Enhanced Raman Spectroscopy. ACS Nano 2011, 5, 307.

13. Chu, Y.; Banaee, M. G.; Crozier, K. B. Double-Resonance Plasmon Substrates for Surface-Enhanced Raman Scattering with Enhancement at Excitation and Stokes Frequencies. ACS Nano 2010, 4, 2804.

14. Cho, W. J.; Kim, Y.; Kim, J. K. Ultrahigh-Density Array of Silver Nanoclusters for SERS Substrate with High Sensitivity and Excellent Reproducibility. ACS Nano 2012, 6, 249.

15. Kaminow, I. P.; Mammel, W. L.; Weber, H. P. Metal-Clad Optical Waveguides: Analytical and Experimental Study. Appl. Opt. 1974, 13, 396.

16. Todorov, Y.; Tosetto, L.; Teissier, J.; Andrews, A. M.; Klang; Colombelli, R.; Sagnes, I.; Strasser, G.; Sirtori, C. Optical Properties of Metal-Dielectric-Metal Microcavities in the THz Frequency Range. Opt. Express 2010, 18, 13886.

17. Engheta, N.; Salandrino, A.; Alù, A. Circuit Elements at Optical Frequencies: Nanoinductors, Nanocapacitors, and Nanoresistors. Phys. Rev. Lett. 2005, 95, 095504.

18. Zhou, J.; Economon, E. N.; Koschny, T.; Soukoulis, C. M. Unifying Approach to Left-Handed Material Design. Opt. Lett. 2006, 31, 3620.

19. Fu, L.; Schweizer, H.; Guo, H.; Liu, N.; Giessen, H. Synthesis of Transmission Line Models for Metamaterial Slabs at Optical Frequencies. Phys. Rev. B 2008, 78, 115110.

20. Chu, Y.; Wang, D.; Zhu, W.; Crozier, K. B. Double Resonance Surface Enhanced Raman Scattering Substrates: An Intuitive Coupled Oscillator Model. Opt. Express 2011, 19, 14919.

21. $\mathrm{Pu}, \mathrm{M} . ; \mathrm{Hu}, \mathrm{C} . ;$ Wang, M.; Huang, C.; Zhao, Z.; Wang, C.; Feng, Q.; Luo, X. Design Principles for Infrared Wide-Angle Perfect Absorber Based on Plasmonic Structure. Opt. Express 2011, 19, 17413.

22. Miyazaki, H.; Kurokawa, Y. Squeezing Visible Light Waves into a 3-nm-Thick and 55-nm-Long Plasmon Cavity. Phys. Rev. Lett. 2006, 96, 97401.

23. Liu, H.; Liu, Y. M.; Li, T.; Wang, S. M.; Zhu, S. N.; Zhang, X. Coupled Magnetic Plasmons in Metamaterials. Phys. Status Solidi B 2009, 246, 1397.

24. Yariv, A.; Xu, Y.; Lee, R. K.; Scherer, A. Coupled-Resonator Optical Waveguide: A Proposal and Analysis. Opt. Lett. 1999, 24, 711.

25. Grigorenko, A. N.; Geim, A. K.; Gleeson, H. F.; Zhang, Y.; Firsov, A. A.; Khrushchev, I. Y.; Petrovic, J. Nanofabricated Media with Negative Permeability at Visible Frequencies. Nature 2005, 438, 335.

26. Giannini, V.; Vecchi, G.; Gómez Rivas, J. Lighting Up Multipolar Surface Plasmon Polaritons by Collective Resonances in Arrays of Nanoantennas. Phys. Rev. Lett. 2010, 105, 266801.

27. Auguié, B.; Barnes, W. Collective Resonances in Gold Nanoparticle Arrays. Phys. Rev. Lett. 2008, 101, 143902.

28. Feigenbaum, E.; Atwater, H. A. Resonant Guided Wave Networks. Phys. Rev. Lett. 2010, 104, 147402

29. Aydin, K.; Ferry, V. E.; Briggs, R. M.; Atwater, H. A. Broadband Polarization-Independent Resonant Light Absorption Using Ultrathin Plasmonic Super Absorbers. Nat. Commun. 2011, 2, 517 . 
30. Wanga, S.; Shana, X.; Patela, U.; Huanga, X.; Lua, J.; Lid, J.; Taoa, N. Label-Free Imaging, Detection, and Mass Measurement of Single Viruses by Surface Plasmon Resonance. Proc. Natl. Acad. Sci. U.S.A. 2010, 107, 16028.

31. Liu, N.; Tang, M. L.; Hentschel, M.; Giessen, H.; Alivisatos, A. P. Nanoantenna-Enhanced Gas Sensing in a Single Tailored Nanofocus. Nat. Mater. 2011, 10, 631.

32. Moharam, M. G.; Gaylord, T. K. Rigorous Coupled-Wave Analysis of Metallic Surface-Relief Gratings. J. Opt. Soc. Am. A 1986, 3, 1780.

33. Chao, Y.; Tseng, H.; Chang, K.; Chang, C. Three Types of Couplings between Asymmetric Plasmonic Dimers. Opt. Express 2012, 20, 2887. 